\title{
Lectin Histochemistry for Glycoconjugates in the Small Intestines of Piglets Naturally Infected with Isospora suis
}

\author{
Bo-Young $\mathrm{CHOI}^{1)}$, Yong-Sung $\mathrm{SOHN}^{1)}$, Changsun $\mathrm{CHOI}^{1)}$ and Chanhee $\mathrm{CHAE}^{1) *}$ \\ ${ }^{1)}$ Department of Veterinary Pathology, College of Veterinary Medicine and School of Agricultural Biotechnology, Seoul National \\ University, Kwanak-Gu 151-742, Seoul, Republic of Korea
}

(Received 4 June 2002/Accepted 26 November 2002)

ABSTRACT. Composition of glycoconjugates was examined in small intestines naturally infected with Isospora suis in preweaned pigs by use of 21 biotinylated-labeled lectins with avidin-biotin-peroxidase complex method. As compared with control pig, staining of 18 lectins altered in jejunal villus brush border and goblet cells of pigs naturally infected with $I$. suis. These results indicate that $I$. suis infection alters carbohydrate residues on the jejunal intestines.

KEY WORDS: histochemistry, Isospora suis, lectin.

Coccidia are a common cause of diarrhea in most mammalian and avian species $[3,11]$. Coccidiosis in nursing pigs is caused by the intracellular protozoan parasite, Isospora suis. Clinical signs in affected pigs include yellowish to greyish diarrhea, dehydration, and weight loss in 5- to 14day old piglets with high morbidity and low mortality $[3,12$, 13]. Escherichia coli was frequently isolated from the small intestines of the coccidially infected piglets [3, 7, 11]. Eimeria tenella and Echinostoma caproni infection altered glycoconjugates composition in intestinal mucosa and may facilitate bacterial adherence to intestinal brush border [1, 9]. Therefore, I. suis infection may affect host intestines and allow newly introduced $E$. coli to colonize in the intestine. However, the effect of I. suis infection on expression of jejunal glycoconjugates has not been investigated yet. The objective of the present study was to determine the alteration of glycoconjugate in the jejunum of pigs naturally infected with $I$. suis by lectin histochemistry.

Twenty 7-day-old Landrace-Large White cross-bred piglets naturally infected with $I$. suis were used in this study. All piglets were tested by histopathologic examination, culture, immunofluorescence, and polymerase chain reaction for transmissible gastroenteritis virus, porcine epidemic diarrhea virus, rotavirus, and E. coli. Ten 7-day-old conventional Landrace-Large White cross-bred pigs selected from five minimal disease herds were used as negative control. Segments of intestines were obtained from middle portion of the jejunum. The samples were fixed with neutral buffered $10 \%$ formalin. After fixation overnight, tissues were processed for paraffin embedding, sectioned at $3 \mu \mathrm{m}$, and mounted on glass slides.

Tissue sections were stained for lectin binding sites using a modification of the avidin-biotin-peroxidase complex (ABC) method as previously described $[2,4,10]$. All procedures were performed at room temperature. Serial sec-

\footnotetext{
* Correspondence to: Chae, C., Department of Veterinary Pathology, College of Veterinary Medicine, Seoul National University, Kwanak-Gu, 151-742, Seoul, Republic of Korea.
}

tions were deparaffinized in xylene, hydrated through a graded series of alcohols to distilled water. Endogenous peroxidase was quenched with absolute methanol containing $0.3 \%$ hydrogen peroxide for $15 \mathrm{~min}$. Each section was treated for $1 \mathrm{hr}$ with one of 21 biotinylated lectins (Vector Laboratories, Burlingame, CA, U.S.A.) at a concentration of $10 \mu \mathrm{g} / \mathrm{m} l$ in phosphate buffered saline (PBS, $\mathrm{pH} 7.2$ ). The lectins used, their acronyms, and major specific sugars are summarized in Table 1. The sections were washed three times with PBS and incubated for $1 \mathrm{hr}$ with an avidin-biotinperoxidase mixture (ABC Kit, Vector Laboratories). The sections were washed three times in PBS, and the final reaction product was produced by immersing the sections in a solution of $0.01 \%$ hydrogen peroxide and $0.05 \%$ 3,3'-diaminobenzidine tetrahydrochloride (DAB) in PBS for $15 \mathrm{~min}$. The sections were lightly counterstained with Mayer's hematoxylin, dehydrated through graded concentrations of ethanol and xylene, and mounted. Control tissues were processed without lectin.

E. coli was isolated from 12 pigs naturally infected with I. suis. Among 12 E. coli, 7 carried gene for F6, 2 carried gene for F4, 1 carried gene for F41. Two islolates did not carried any fimbrial or enterotoxin genes. Immunofluorescent tests for porcine epidemic diarrhea virus, transmissible gastroenteritis virus, and rotavirus were negative in the small intestines of all pigs used in this study.

Histopathological lesions in the jejunum of all affected piglets were similar. Villi showed various degrees of atrophy associated with changes in their apical epithelium. Coccidial organisms were present in variable numbers of the cells lining the villi and were mainly asexual stages (meronts and merozoites). Sexual stages were also present in some cases and identified as spherical or oval macrogamonts in different stages of maturity.

At the lectin concentration used, labeling was specific and reproducible; and non-specific background staining was minimal or absent. Lectin histochemical characteristics of jejunum are summarized in Table 1 . The villi tip was used 
Table 1. Lectin-binding pattern in the brush border of jejunal enterocytes from twenty 7-day-old piglets naturally infected Isospora suis and 10 control piglets

\begin{tabular}{|c|c|c|c|c|c|c|}
\hline \multirow[t]{2}{*}{ Lectin $^{a)}$} & \multirow[t]{2}{*}{ Abbreviation } & \multirow{2}{*}{$\begin{array}{l}\text { Binding } \\
\text { specificity }\end{array}$} & \multicolumn{2}{|c|}{ Control } & \multicolumn{2}{|c|}{ I. suis } \\
\hline & & & Enterocyte & Goblet cell & Enterocyte & Gobletcell \\
\hline \multicolumn{7}{|l|}{$\mathrm{N}$-acetylgalactosamine group: } \\
\hline Bandeiraea simplicifolia lectin I & BSL I & $\alpha$-GalNAc, $\alpha$-Gal & $++^{\mathrm{b})}$ & + & + & + \\
\hline Dolichos biflorus agglutinin & DBA & $\alpha$-GalNAc & + & - & + & - \\
\hline Glycine maxi (soybean agglutinin) & SBA & $\alpha$-GalNAc & ++ & + & + & + \\
\hline Ricinus communis agglutinin I & RCA I & $\beta$-GalNAc, $\beta$-Gal & ++ & ++ & ++ & +++ \\
\hline Sophora japonica agglutinin & SJA & $\beta$-GalNAc & + & + & + & + \\
\hline Vicia villosa agglutinin & VVA & $\beta$-GalNAc & + & - & + & - \\
\hline \multicolumn{7}{|l|}{$\mathrm{N}$-acetylglucosamine group: } \\
\hline Bandeiraea simplicifolia lectin II & BSL II & $\alpha-, \beta$-GlcNAc & - & - & - & - \\
\hline Datura stramonium lectin & DSL & $\beta$-GlcNAc & ++ & + & ++ & + \\
\hline Lycopersicon esculentum lectin & LEL & $\beta$-GlcNAc & +++ & - & + & - \\
\hline Solanum tuberosum lectin & STL & $\beta$-GlcNAc & ++ & - & + & - \\
\hline Triticum vulgaris (wheat germ) & WGA & $\beta$-GlcNAc & ++ & +++ & ++ & +++ \\
\hline Succinylated Triticum vulgaris & s-WGA & $\beta$-GlcNAc & - & - & - & - \\
\hline \multicolumn{7}{|l|}{ Galactose group: } \\
\hline Arachis hypogaea (peanut) & PNA & $\beta$-Gal & ++ & - & + & - \\
\hline Artocarpus integrifolia (Jacalin) & Jacalin & $\beta$-Gal & +++ & ++ & +++ & +++ \\
\hline Erythrina crisagalli lectin & ECL & $\beta$-Gal $>\beta$-GalNAc & +++ & +++ & +++ & ++ \\
\hline \multicolumn{7}{|l|}{ Glucose/mannose group: } \\
\hline Canavalia ensiformis (concanavalin A) & ConA & $\alpha-$ Man $>\alpha$-Glc & ++ & + & +++ & ++ \\
\hline Pisum sativum agglutinin & PSA & $\alpha-\operatorname{Man}>\alpha$-Glc & - & + & +++ & + \\
\hline Lens culinaris agglutinin & LCA & $\alpha-\operatorname{Man}>\alpha-\mathrm{Glc}$ & + & + & + & + \\
\hline \multicolumn{7}{|l|}{ Fucose group: } \\
\hline Ulex europaeus-I & UEA-I & $\alpha$-Fuc & + & ++ & + & + \\
\hline \multicolumn{7}{|l|}{ Oligosaccharide group: } \\
\hline Phaseolus vulagaris agglutinin-E & PHA-E & oligosaccharide & +++ & +++ & +++ & +++ \\
\hline Phaseolus vulagaris agglutinin-L & PHA-L & oligosaccharide & + & + & + & + \\
\hline
\end{tabular}

a) Lectins are divided into 6 groups depending on their binding specificity.

b) $-=$ negative staining, $+=$ faint staining, $++=$ moderate staining, $+++=$ strong staining.

to determine the degree of staining for villus brush border. The positive staining reaction was recognized by the occurrence of brown reaction products on the cell membrane. Staining intensity of lectins were increased in jejunal villus brush border of pigs naturally infected with $I$. suis compared with control pigs, i. e., Pisum sativum agglutinin (PSA) (Figs. 1, 2) and concanavalin A (ConA), whereas staining intensity of lectins are decreased i. e., Bandeiraea simplicifolia lectin I (BSL I), soybean agglutinin (SBA), Lycopersicon esculentum lectin (LEL) (Figs. 3, 4), Solanum tuberosum lectin (STL), peanut agglutinin (PNA), and Lens culinaris agglutinin (LCA). Staining intensity of lectins are increased in jejunal goblet cells of pigs naturally infected with I. suis compared with control pigs, i. e., Ricinus communis agglutinin I (RCA I), ConA, and Jacalin whereas staining intensity of lectins are decreased i. e., Datura stramonium lectin (DSL), Erythrina crisagalli lectin (ECL), and Ulex europaeus-I (UEA-I).

The effect of I. suis infection on expression of jejunal glycoconjugates has not been previously investigated. Lectins were used as histochemical probes to analyze the change in the expression of glycoconjugate of proximal small intestine in response to $I$. suis infection. These results indicate that $I$. suis infection alters carbohydrate residues on the jejunal villus enterocytes. The binding patterns in the control 7-day- old pig were in general agreement with those of a previous study [4].

Mucus acts as a barrier which protects a host against colonization by microbial pathogens. Microorganisms must have adherence to colonize in the intestine. Trapping of microorganisms within mucus is considered important because it may prevent adherence of bacteria to intestinal epithelial cells $[6,8]$. Alteration of the intestinal glycoconjugates affects bacterial adherence to the mucosal surface. For example, coccidial infection may induce an increase of mannose residues on the intestinal surface and allow adhesion of more Salmonella Typhimurium to the intestine in poultry [1]. The significant increased reactivity of jejunum from I. sius-infected pigs as compared with those from normal pigs to glucose/mannose-specific lectin such as PSA implicates this glycoconjugate as a potential factor in the enhancement of intestinal colonization by E. coli following I. suis infection. It has been reported that porcine enterotoxigenic $E$. coli binds specifically to PSA [5, 14, 15]. The increased staining intensity of PSA that has high affinity for E. coli indicated that altered composition of glycoconjugates in pigs naturally infected with $I$. suis promote intestinal colonization by serving as a site for the adherence of $E$. coli strains. Therefore, alteration in the composition of glycoconjugates as the result of $I$. suis infection could be possi- 

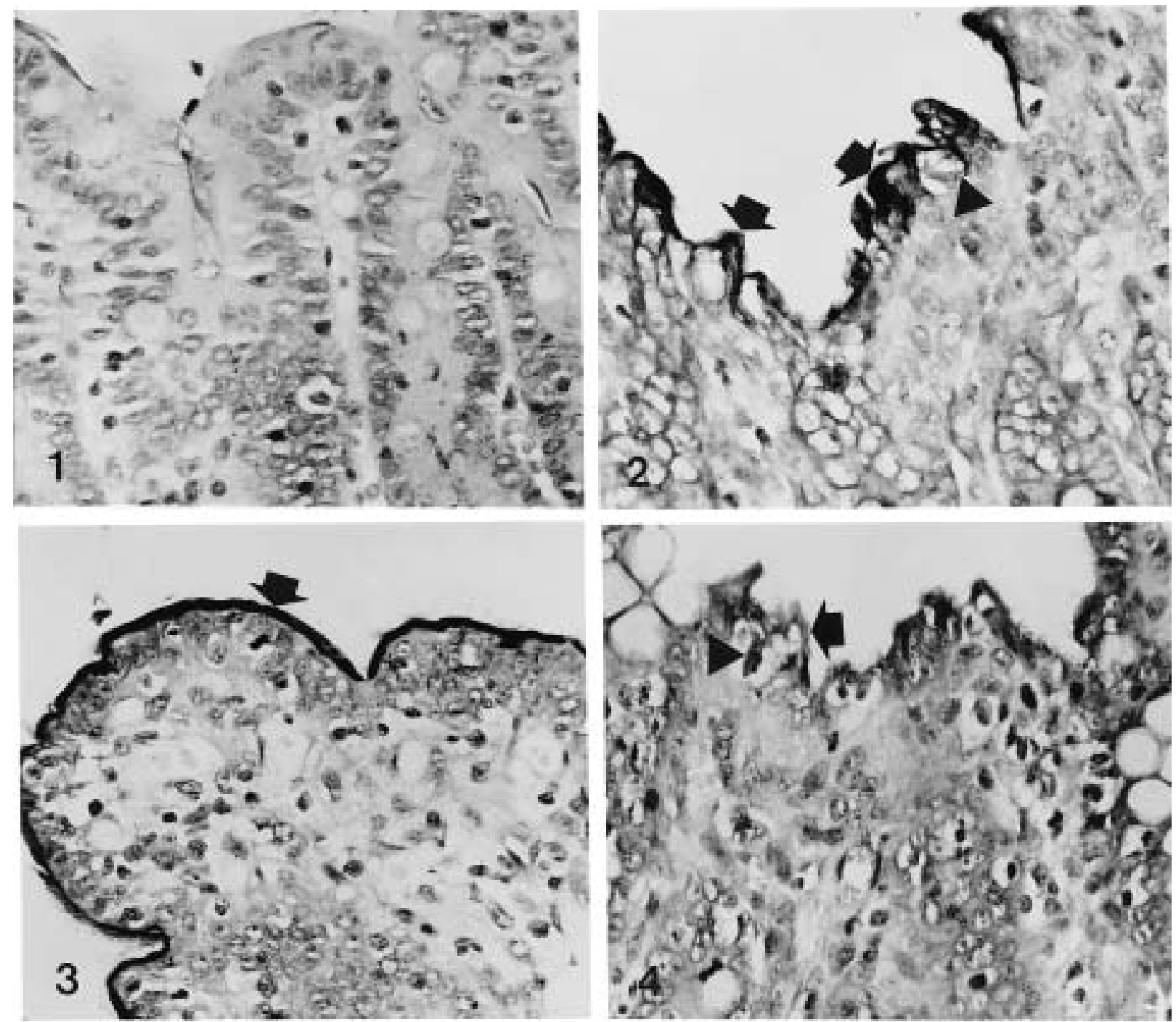

Fig. 1. Jejunum from a 7-day-old pig. Notice negative lectin affinity for brush border of enterocytes and goblet cells. PSA staining, ABC, $\mathrm{H}_{2} \mathrm{O}$, DAB, Mayer's hematoxylin counterstain, $\times 400$.

Fig. 2. Jejunum from a 7-day-old pig naturally infected with Isospora suis. Notice strong lectin affinity for brush border of enterocytes (arrows) and meronts (arrow head) of $I$. suis within villus enterocyte. PSA staining, ABC, $\mathrm{H}_{2} \mathrm{O}$, DAB, Mayer's hematoxylin counterstain, $\times 400$.

Fig. 3. Jejunum from a 7-day-old pig. Notice strong lectin affinity for brush border of enterocytes (arrow). LEL staining, $\mathrm{ABC}, \mathrm{H}_{2} \mathrm{O}$, DAB, Mayer's hematoxylin counterstain, $\times 400$.

Fig. 4. Jejunum from a 7-day-old pig naturally infected with I. suis. Notice weak lectin affinity for brush border of enterocytes (arrow) and meronts (arrow head) of I. suis within villus enterocyte. LEL staining, ABC, $\mathrm{H}_{2} \mathrm{O}, \mathrm{DAB}$, Mayer's hematoxylin counterstain, $\times 400$.

ble factor that predisposes pig to enhance $E$. coli infection in the small intestine.

ACKNOWLEDGMENT. This study was supported by contract research fund of Research Institute for Veterinary Science of College of Veterinary Medicine, Seoul National University and Brain Korea 21 Project in the Republic of Korea.

\section{REFERENCES}

1. Baba, E., Tsukamoto, Y., Fukata, T., Sasai, K. and Arakawa, A. 1993. Am. J. Vet. Res. 54: 1471-1475.

2. Chae, C. 1997. J. Vet. Med. Sci. 59: 931-934.

3. Chae, C., Kwon, D., Kim, O., Min, K., Cheon, D.-S., Choi, C., Kim, B. and Suh, J. 1998. Vet. Rec. 143: 417-420.

4. Chae, C. and Lee, Y.-S. 1995. J. Vet. Med. Sci. 57: 883-889.

5. Dean, E. A. and Isaacson, R. E. 1985. Infect. Immun. 47: 98 105. 
6. Drumm, B., Robertson, A. M. and Sherman, P. M. 1988. Infect. Immun. 56: 2437-2442.

7. Eustis, S. L. and Nelson, D. T. 1981. Vet. Pathol. 18: 21-28.

8. Forstner, J. F. 1978. Digestion 17: 234-263.

9. Fujino, T. and Fried, B. 1993. J. Helminthol. 67: 179-188.

10. Jeong, K.-I., Sohn, Y.-S., Ahn, K., Choi, C, Han D. U. and Chae, C. 2002. J. Vet. Med. Sci. 64: 535-538.

11. Johnson, M. W., Fitzgerald, G. R., Welter, M. W. and Welter,
C. J. 1992. Vet. Med. 87: 382-386.

12. Lindsay, D. S. 1989. Vet. Med. 84: 443-448.

13. Lindsay, D. S., Blagburn, B. L. and Powe, T. A. 1992. Compend. Contin. Educ. Pract. Vet. 14: 698-702.

14. Smyth, C. J., Marron, M. and Smith, S. G. J. 1994. In: Escherichia coli in Domestic Animals and Humans. CAB International, Oxon.

15. Sohn Y.-S. and Chae, C. 2000. J. Vet. Med. Sci. 62: 543-547. 